\title{
Statistical Calculations of the Accuracy of the Michaelis Constant From Viscosimetric Determinations of Polymetaphosphatase and Dextranase Activity
}

\author{
ESK I L H UL T I N*
}

Department of Pharmacology, UCLA School of Medicine, Los Angeles, Calif. 90024, U.S.A.

\begin{abstract}
Values for Michaelis' constant obtained with different preparations of polymeric homologous substrates can be compared by standard statistical methods if the accuracy is known, but the most common procedures for the calculation of Michaelis' constant are not suitable for statistical accuracy calculations according to the method of least squares; however, Hanes' equation $[\mathrm{S}] / v=K_{\mathrm{m}} / V+(1 / V) \cdot[\mathrm{S}]$ is suitable. The use of this equation for accuracy calculations is exemplified.

For Aspergillus niger polymetaphosphatase the values of Michaelis' constant showed no significant difference for three preparations of polymetaphosphate, whereas a fourth preparation with a larger fraction of low molecular weight substance gave a deviating result. The $80 \%$ confidence interval for the weighted mean value of the Michaelis' constant for the three preparations was $0.06-0.13 \%$ at $25^{\circ} \mathrm{C}$ and $\mathrm{pH}$ 5.4 in acetate buffer of the ionic strength 0.3 .

For Cellvibrio fulva dextranase the $95 \%$ confidence interval for a determination of the Michaelis' constant was $0.1-0.6 \%$ at $25^{\circ} \mathrm{C}$ and $\mathrm{pH}$ 5.22; the $50 \%$ confidence interval was $0.31-0.40 \%$.
\end{abstract}

Dolymetaphosphatase and dextranase are examples of enzymes which can act as catalysts for the hydrolysis of polymeric homologous substances which give rather viscous solutions. Because the viscosity depends not only on the concentration but also on the degree of polymerization of the substrate, and because viscosimetric measurements can be done rather accurately, viscosimetric methods are particularly suitable for the determination of enzyme activities which are so low that stoichiometric methods could hardly be used.

* On leave of absence from the University of Stockholm, Sweden. Present address: Biokemiska institutionen, Stockholms universitetet, Stockholm Va, Sweden. 


\section{THEORETICAL}

Viscosity, concentration, and degree of polymerization. Unfortunately, most viscosimetric methods do not give an unbiased estimate of the number of molecules which have reacted. However, it has been shown mathematically by the present author that if the enzyme attacks all linkages between the primary units of the polymeric substrate with equal ease, and if the viscosity of the polymeric homologous substrate follows Staudinger's equation ${ }^{1}$ (which most substances do only approximately), ${ }^{2}$ it is possible to obtain from viscosimetric measurements a value for enzymatic activity, proportional to the number of linkages broken. For this calculation the inverse value of the specific viscosity is plotted in a graph versus time, a straight line is fitted, and the inclination of this line is multiplied with the square of the substrate concentration. ${ }^{3-6}$

A prerequisite is that the molecular weight distribution of the polymer preparation used for substrate is the same as the distribution obtained if an infinitely large molecule were broken down to the same average degree of polymerization. ${ }^{7,8}$ Substrates actually available cannot generally be expected to have ideal qualities, and so some deviations from the simple theory may be expected to occur in actual viscosimetric determinations of enzymic activity, but if the ranges of depolymerization in different enzyme experiments are not much apart, the deviations can be expected to be negligible.

Substrate concentration and enzymic activity. The influence of the substrate concentration on the viscosity and on the viscosity changes of the reaction mixture can thus easily be eliminated. However, the substrate concentration has an influence on the enzymic activity: according to the theory of Henri, enzyme molecules are active only if they are part of an enzyme-substrate complex formed by the principle of the law of mass action. ${ }^{9,10}$ This influence of the substrate concentration, usually expressed as the value of Michaelis' constant for the particular enzymic reaction, has been exemplified for viscosimetric measurements with the determination of the value of Michaelis' constant for trypsin. ${ }^{11}$

It is nowadays a standard procedure to calculate a value for Michaelis' constant according to either of three simple linear equations which were originally suggested by Hanes, ${ }^{12}$ by Lineweaver and Burk, ${ }^{13}$ and by Eadie. ${ }^{14}$ The value is usually obtained from a graph. The accuracy of the value is rarely considered or calculated, although the accuracy may be essential for the comparison of results from different experimental series.

Conditions for use of the method of least squares. The accuracy of values obtained for Michaelis' constant is easily calculated if the conditions for using the method of least squares are satisfied. These conditions require that the independent variable in the calculation must be actually independent, and that the accuracy of the dependent variable must be the same for all values of the independent variable. These conditions are not satisfied for two of the common methods for the calculation of Michaelis' constant but approximately satisfied for the remaining one, as first pointed out by Hultin and Lundblad and later also by Wilkinson.11,15 
The most commonly used method is the one introduced by Lineweaver and Burk in which the inverse value of the substrate concentration is the independent variable, and the inverse value of the enzymic activity is the dependent variable. If the absolute (and not the relative) accuracy by which the enzymic activity is measured, is independent of its value, the accuracy of its inverse value decreases considerably and consistently as the inverse value of the substrate concentration increases; consequently the method of least squares cannot be used. Furthermore, it cannot be used if the calculation is done according to the method of Eadie in which the enzymic activity is considered to be a function of the quotient between the enzymic activity and the substrate concentration; this quotient is not an actually independent variable because the enzyme activity which is an actually dependent variable, is included. However, the conditions for using the method of least squares are approximately satisfied for Hanes' method in which the quotient between the substrate concentration and the enzymic activity is considered to be a function of the substrate concentration. Consequently, the method of least squares can be used with Hanes' equation for the calculation of the value of Michaelis' constant and the accuracy of this determination.

Hanes' equation can be written as follows:

$$
\frac{[\mathrm{S}]}{v}=\frac{K_{\mathrm{m}}}{V}+\frac{1}{V} \cdot[\mathrm{S}]
$$

where $K_{\mathrm{m}}$ is Michaelis' constant, [S] is the substrate concentration, $v$ is the enzymic acitivity measured, and $V$ is the limit value for enzymic activity at increasing substrate concentration. In this equation, $[\mathrm{S}]$ is the independent variable, and the quotient $[\mathrm{S}] / v$ is the dependent variable. The value of Michaelis' constant is calculated as the value of the independent variable (the abscissa value), with reversed sign, for which the value of the dependent variable (the ordinata value) is zero. The procedure for the calculation of the standard error for such a value according to the method of least squares has been adequately described in the literature, and so the details are not given here. ${ }^{16-18}$

\section{EXPERIMENTAL AND RESULTS}

Polymetaphosphatase. Several molds contain enzymes which can degrade polymetaphosphate, and Ingelman and Malmgren investigated the possibility of measuring polymetaphosphatase activity viscosimetrically. ${ }^{19}$ They used preparations from Aspergillus niger. In experiments with different enzyme concentrations but equal substrate concentrations the enzyme activities observed agreed rather well with those expected. However, comparatively small changes in ionic strength had such a great effect that the introduction of an ionic factor was suggested. In experiments with various substrate concentrations and several different preparations of polymetaphosphate, agreement was not perfect between the activities expected and those obtained by multiplying the square of the substrate concentrations $c$ with the inclination of lines fitted to the plots of the viscosimetric measurements; however, Ingelman and Malmgren found that agreement would be attained if the inclination of the 


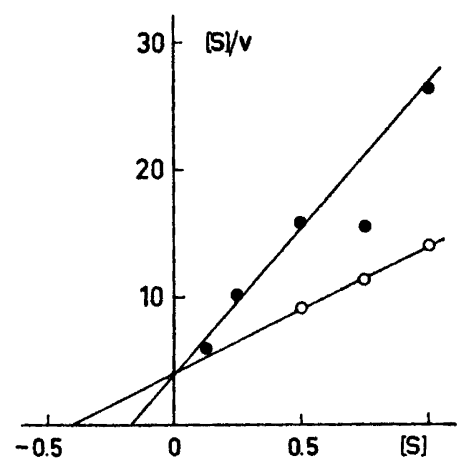

Fig. 1. Graph for the calculation of the Michaelis' constant $K_{\mathrm{m}}$ of mold polymetaphosphatase preparations K 10 (dots; a misprint (possibly 860 instead of 680) presumably causes the strong deviation of one point), and $K 12$ (rings).

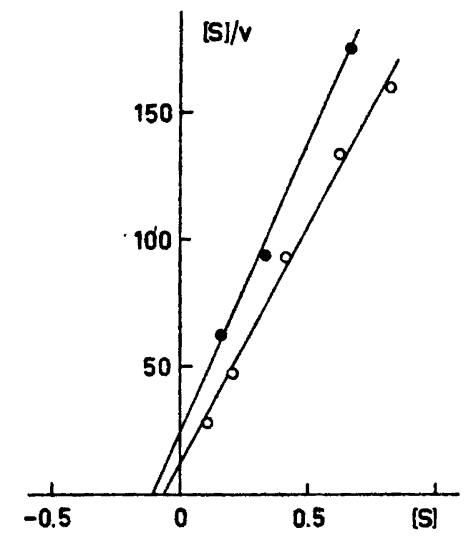

Fig. 2. Graph for the calculation of the Michaelis' constant $K_{m}$ of mold poly. metaphosphatase for the polymetaphos. phate preparations $K 11$ (dots) and $K 15$ (rings).

lines were multiplied by $c^{1.71}$. This suggestion for treatment of the experimental results implies ignoring Henri's theory concerning the influence of substrate concentration on enzymic activity and the introduction of a curve-fitting technique without theoretical basis.

The results from one of the preparations differed from those obtained with the others; this preparation was assumed to contain a fraction of relatively low molecular weight polymetaphosphate, which is against the presumptions for the deduction of the expression for enzymic activity. ${ }^{3,8}$

The values given for the concentration of the polymetaphosphate solutions and the inclinations of the lines fitted to the viscosimetric measurements can be used for the calculation of the Michaelis constant for the Aspergillus niger polymetaphosphatase at the experimental conditions used $\left(25^{\circ} \mathrm{C}\right.$ and $\mathrm{pH} 5.4$ in acetate buffer of ionic strength 0.3 ). The graphs corresponding to the calculations according to the method of least squares are shown as Figs. 1 and 2. The intersection between the abscissa and a straight line fitted to the plots of the experimental values gives the negative value of the Michaelis' constant. This value was calculated, and also the corresponding standard error and the $80 \%$ confidence interval, using the appropriate value of the $t$-distribution. ${ }^{20}$ The $80 \%$ confidence interval was chosen instead of the $95 \%$ interval (one of the customary intervals in statistical calculations) because the lower fiducial limit would have reached 0 for a lower confidence than $95 \%$ for preparations $\mathrm{K} 11$ and $\mathrm{K} 15$. The results are given in Table 1 . It is evident that the results from three of the four substrate preparations are statistically in very good agreement; the deviating preparation is the one which is supposed to contain a large fraction of low molecular weight polymetaphosphate. The values 
Table 1. The Michaelis' constant $K_{\mathrm{m}}$ for mold polymetaphosphatase, using four preparations of polymetaphosphate. ${ }^{10}$ The concentrations are in per cent. For preparation $\mathrm{K} 10$ the calculations were carried out without the deviating measurement (see Fig. 2).

\begin{tabular}{|c|c|c|c|c|c|}
\hline Preparation & $K_{\mathrm{m}}, \%$ & $\begin{array}{l}\text { Standard } \\
\text { error }\end{array}$ & $\begin{array}{l}\text { Degrees of } \\
\text { freedom }\end{array}$ & \multicolumn{2}{|c|}{$\begin{array}{l}80 \% \text { fiducial } \\
\text { limits for } K_{m}\end{array}$} \\
\hline K 10 & 0.17 & 0.04 & 2 & 0.10 & 0.24 \\
\hline K 11 & 0.10 & 0.02 & 1 & 0.04 & 0.16 \\
\hline K 12 & 0.40 & 0.04 & 1 & 0.29 & 0.52 \\
\hline K 15 & 0.06 & 0.03 & 3 & 0.01 & 0.11 \\
\hline
\end{tabular}

obtained from the three preparations were used for the calculation of the weighted mean value of Michaelis' constant and its $80 \%$ confidence interval; the inverse value of the square of the confidence intervals were used as weights. The lower and upper $80 \%$ fiducial limits for the substrate constant of the polymetaphosphatase were $0.06 \%$ and $0.13 \%$.

Dextranase. It was shown by Ingelman that the bacterium Cellvibrio fulva contains dextranase. ${ }^{21}$ First, he tested the applicability of the viscosimetric theory by measuring the effect of various concentrations of this dextranase on the viscosity of dextran solutions at constant substrate concentration. The inverse value of the specific viscosity was plotted versus time for each experiment, and a straight line was obtained. This indicates that the dextranase hydrolyzes all or almost all of the glucosidic linkages with equal ease and that it is an endoenzyme. Furthermore, the inclinations of the lines were proportional to the enzyme concentrations. However, in experiments with different substrate concentrations but equal enzyme concentrations, the results calculated according to the theory, using the square of the substrate concentration $c$ as a factor, were not entirely in agreement. Ingleman noticed that if $c^{1.7}$ instead of $c^{2}$ were used as a factor, the results would be accordant, but in so doing, he was overlooking the influence of the substrate concentration on the enzymic activity.

The values given by Ingelman for the concentration of the dextran solutions and for the inclination of the regression lines were used in the present investigation for the calculation of the Micahelis' constant for the Cellvibrio

Fig. 3. Graph corresponding to the calculation according to Hanes ${ }^{12}$ of the Michaelis' constant $K_{\mathrm{m}}$ of bacterial dextranase. The intersection of the regression line with the axis of abscissas gives the negative value of the substrate constant. The $50 \%$ and the $95 \%$ confidence intervals of this intersection are shown in the figure. The concentration was measured in per cent and the time in minutes.

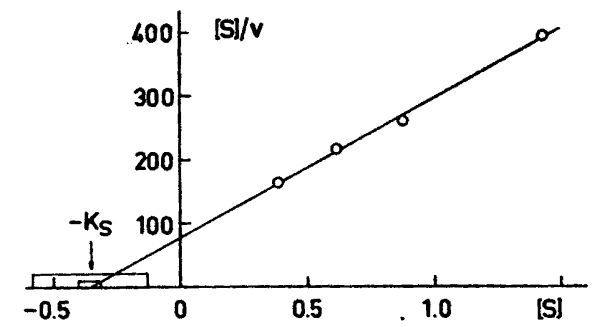


fulva dextranase at $\mathrm{pH} 5.22$ and $25^{\circ} \mathrm{C}$, by means of the method of least squares. As a result, the value of the constant is $0.36 \%$, and the corresponding standard error is $\pm 0.05 \%$. Only 4 measurements were done. Hence, the number of degrees of freedom is 2 , and using the appropriate value of the $t$-distribution as a factor for the standard error, we can calculate confidence intervals. Thus, e.g., the $95 \%$ confidence interval is \pm 0.23 , and the lower and upper $95 \%$ fiducial limits are $0.13 \%$ and $0.58 \%$, respectively. The $50 \%$ fiducial limits are 0.31 and $0.40 \%$, respectively. A corresponding graph is shown as Fig. 3 .

It may be mentioned that another dextranase has been described by Hultin and Nordström. ${ }^{22}$

\section{DISCUSSION}

It is evident from a statistical point of view that for increased accuracy in the determination of substrate constants a larger number of measurements would be desirable, and also measurements at lower substrate concentrations. Even a small increase in the number of experiments in each series, would have given a much more favourable $t$-value for the calculation of the $95 \%$ confidence interval, and a few experiments added, at lower substrate concentrations, would have given a noticeably smaller value for the standard error.

\section{REFERENCES}

1. Staudinger, H. and Heuer, W. Ber. 63 (1930) 222.

2, Mark, H. Der feste Körper, Hirzel, Leipzig 1938, p. 103.

3. Hultin, E. Svensk Kem. Tidskr. 58 (1946) 281.

4. Hultin, E. Acta Chem. Scand. 3 (1949) 625.

5. Hultin, E. and Wanntorp, I. Acta Chem. Scand. 20 (1966) 2667.

6. Lundblad, G. and Hultin, E. Scand. J. Clin. Lab. Invest. 18 (1966) 201.

7. Sillén, L. G. Svensk Kem. Tidskr. 55 (1943) 221.

8. Hultin, E. J. Polymer Sci. 23 (1957) 103.

9. Henri, V. Compt. Rend. 135 (1902) 916.

10. Henri, V. Arch. Fisiol. 1 (1904) 299.

11. Hultin, E. and Lundblad, G. Acta Chem. Scand. 9 (1955) 1610.

12. Hanes, C. S. Biochem. J. 26 (1932) 1406.

13. Lineweaver, H. and Burk, D. J. Am. Chem. Soc. 56 (1934) 658.

14. Eadie, G. S. J. Biol. Chem. 146 (1942) 85.

15. Wilkinson, G. N. Biochem. J. 80 (1961) 324.

16. Bennett, C. A. and Franklin, N. L. Statistical Analysis in Chemistry and the Chemical Industry, Wiley, New York 1954, p. 231.

17. Davies, O. L. Statistical Methods in Research and Production, 3rd. Ed., Oliver and Boyd, London 1957, p. 170.

18. Brownlee, K. A. Statistical Theory and Methodology in Science and Engineering, Wiley, New York 1960, p. 284.

19. Ingelman, B. and Malmgren, H. Acta Chem. Scand. 2 (1948) 365.

20. Fisher, R. A. and Yates, F. Statistical Tables for Biological, Agricultural and Medical Research, Oliver and Boyd, London 1953, p. 40.

21. Ingelman, B. Acta Chem. Scand. 2 (1948) 803.

22. Hultin, E. and Nordström, L. Acta Chem. Scand. 3 (1949) 1405.

Received March 13, 1967. 